\title{
The Anatomical Research on the Mental Foramen Related to the Mental Nerve Block
}

\author{
Lanqian Xie ${ }^{1}$, Zhenxing Zhao², Liang Huang2, Chao Qin², Wenjuan Wang" ${ }^{2}$ Cheng $\mathrm{Xu}^{2 *}$ \\ ${ }^{1}$ Department of Nephrology, Wuhan No. 1 Hospital, Wuhan, China \\ ${ }^{2}$ Department of Pain Medicine, Jingzhou First People's Hospital, The First Affiliated Hospital of Yangtze University, Jingzhou, \\ China \\ Email: 31859555@qq.com
}

How to cite this paper: Xie, L.Q., Zhao, Z.X., Huang, L., Qin, C., Wang, W.J. and $\mathrm{Xu}$, C. (2021) The Anatomical Research on the Mental Foramen Related to the Mental Nerve Block. Yangtze Medicine, 5, 54-60. https://doi.org/10.4236/ym.2021.51006

Received: January 24, 2020

Accepted: January 11, 2021

Published: January 14, 2021

Copyright (c) 2021 by author(s) and Scientific Research Publishing Inc. This work is licensed under the Creative Commons Attribution International License (CC BY 4.0).

http://creativecommons.org/licenses/by/4.0/

\section{(c) (i) Open Access}

\begin{abstract}
Objective: The aim of this study was to observe and investigate the location of mental foramen, providing scientific and reasonable anatomic basic for clinical mental nerve block. Methods: The shape, orientations, relationship with teeth and number of accessory mental foramina in 50 (100 side) adult mental foramina were observed. The long and short diameters of the mental foramen and the distance between the mental foramen and the peripheral anatomical markers were measured by a vernier caliper, and statistical analysis of the data was performed to obtain the final results. Results: The probability of mental foramen being elliptical in shape accounted for $27 \%$, circular for $73 \% ; 10 \%$ of the mental foramen openings are outward and $90 \%$ are outward and upward; The mental foramina were located under the first premolars in $20 \%$, under the second premolars in $75 \%$, and $5 \%$ situated in the bottom of the first molars. The incidence of accessory mental foramina was $1 \%$; the long diameter of the mental foramen was $(2.42 \pm 0.71) \mathrm{mm}$, and the short diameter of the mental foramen was $(2.15 \pm 0.14) \mathrm{mm}$; the vertical distance from the mental foramen center to the alveolar crest and the lower margin of the mandible were $(14.09 \pm 3.23) \mathrm{mm}$ and $(14.56 \pm 1.74) \mathrm{mm}$; the distance between the midpoints of the vertical line to the center of the mental foramen was $(1.26 \pm 0.97) \mathrm{mm}$; the horizontal distance from the center of the mental foramen to the anterior median line was $(23.57 \pm 1.82) \mathrm{mm}$. Conclusion: The point of mental nerve block was selected below and behind the second premolars, above the midline of the mandible. The distance from the anterior median line was $2.5 \mathrm{~cm}$, and the mental foramen was explored forward and downward after the insertion.
\end{abstract}

\section{Keywords}

Mental Nerve Block, Mental Foramen 


\section{Introduction}

Mental nerve blocks can treat pains in the area of the mental nerve, including mental nerve injuries, mental nerve entrapments, local cancer pains [1], and herpes zoster pains. Moreover, the nerve block in this part should also be applied to local lesion resections, plastic surgeries, and dental surgeries, etc. With the gradual increase of the applications of mental nerve block, it has attracted more and more clinical attention. Although many scholars at home and abroad have carried out relevant studies on the mental foramen, professional anatomical research on mental nerve block has not been conducted so far. Therefore, it is still difficult to clinically perform a mental nerve block. Hence, this study provides the clinical guidance for mental nerve block by measuring the relevant data of mandible mental foramen and its peripheral anatomical marks in Jianghan Plain.

\section{Materials and Methods}

\subsection{Research Objects}

50 cases (100 sides) of complete adult mandible specimen without developmental abnormalities in Jianghan Plain: provided by Anatomy Laboratory, Department of Basic Medical Sciences, Yangtze University.

\subsection{Measuring Equipment}

Vernier caliper, ruler, wire, No. 7 needle, and camera.

\subsection{Measuring Method}

\subsubsection{Measuring Method}

The mandible specimens that meet the standards were numbered. The data were measured twice with a vernier caliper to take the average; and a video footage was taken.

\subsubsection{Observation of the Shape, Size, and Position of the Mental Foramen}

The long and short diameters of the mental foramen were measured with a vernier caliper. The long and short diameters were deemed the same if the difference between them is smaller than $0.1 \mathrm{~mm}$. The intersection of the long and short diameters is the center of the mental foramen.

\subsubsection{Measurement of the Distance between the Mental Foramen and Peripheral Anatomical Marks}

Vertical and horizontal lines were made at point $\mathrm{A}$ in the center of the mental foramen. The intersection of the vertical line with the alveolar bone crest was Point $\mathrm{B}$ and that with the inferior border of mandible Point $\mathrm{C}$. The midpoint of the line between Points $\mathrm{B}$ and $\mathrm{C}$ was Point $\mathrm{D}$. the intersection of the horizontal line with the anterior median line was Point $\mathrm{E}$. The distances between $\mathrm{AB}, \mathrm{AC}$, $\mathrm{AD}$, and $\mathrm{AE}$ were measured with a vernier caliper. 


\subsection{Statistical Processing}

SPSS 18.0 was used for the statistical processing. The results were expressed as the mean \pm standard deviation $(\chi \pm s)$ and ( $\min -\max )$. The data conforming to the normal distribution were tested by paired sample $t$ test and those non-conforming to the normal distribution by Wilcoxon test. Data on both sides were compared. $\mathrm{P} \leq 0.05$ indicated that the difference had statistical significance.

\section{Results}

\subsection{Shape, Opening Direction, and Position of Mental Foramen and the Occurrence Rate of Submental Foramen}

Among the 50 specimens (100 sides), the numbers (occurrence rate) of left, right oval mental foramen were $13(26 \%)$ and $14(28 \%)$, respectively, and those of circular mental foramen were $37(74 \%)$ and $36(72 \%)$, respectively. The numbers (occurrence rate) of left, right mental foramen with outward opening were 5 $(10 \%)$ and $5(10 \%)$, respectively, and those of mental foramen with outward and upward opening were 45 (90\%) and 45 (90\%). The numbers (occurrence rate) of left, right mental foramen below the first premolar was 10 (20\%) and $10(20 \%)$, respectively, that of mental foramen below the second premolar 38 (76\%) and 37 (74\%), respectively, and that of mental foramen below the first molar $2(4 \%)$ and 3 (6\%), respectively. Among all specimens, there was only 1 case of submental foramen (left); and the occurrence rate was $1 \%$.

\subsection{Measurement and Comparison of Long and Short Diameters of Mental Foramen}

Among the 50 specimens (100 sides), the long diameters of left, right mental foramen were $(2.38 \pm 0.63) \mathrm{mm}$ and $(2.45 \pm 0.79) \mathrm{mm}$, respectively. The short diameters were $(2.13 \pm 0.33) \mathrm{mm}$ and $(2.18 \pm 0.43) \mathrm{mm}$, respectively. The data of left and right long and short diameters were not normally distributed and tested with Wilcoxon rank sum test. There was no statistical difference in the left and right long diameters $(\mathrm{P}=0.42, \mathrm{P}>0.05)$; and there also was no statistical difference in the short diameters $(\mathrm{P}=0.55, \mathrm{P}>0.05)$.

\subsection{Measurement and Comparison of Distance between Mental Foramen and Peripheral Anatomical Marks}

The vertical distance $A B$ between the center of the left mental foramen and the alveolar bone crest was $(14.10 \pm 3.49) \mathrm{mm}(6.15-24.60 \mathrm{~mm})$; the right $\mathrm{AB}$ was $(14.08 \pm 2.98) \mathrm{mm}(7.75-22.10 \mathrm{~mm})$; the $\mathrm{AB}$ of all specimens was $(14.09 \pm 3.23)$ $\mathrm{mm}(6.15-24.60 \mathrm{~mm})$. The left and right data were normally distributed. The paired sample $t$ test showed that there was no statistical difference $(P=0.95, P>$ $0.05)$.

The vertical distance AC between the center of the left mental foramen and inferior border of mandible was $(14.60 \pm 1.75) \mathrm{mm}(11.50-18.50 \mathrm{~mm})$; the right AC was $(14.52 \pm 1.75) \mathrm{mm}(11.15-19.00 \mathrm{~mm})$; the AC of all specimens was $(14.56 \pm 1.74) \mathrm{mm}(11.15-19.00 \mathrm{~mm})$. The left and right data were normally 
distributed. The paired sample t test showed that there was no statistical difference $(\mathrm{P}=0.75, \mathrm{P}>0.05)$.

The absolute value distance $\mathrm{AD}$ between the midpoint of the vertical line of left mandible through the mental foramen to the midpoint of the mental foramen was $(1.29 \pm 1.12) \mathrm{mm}(0.00-4.60 \mathrm{~mm})$; the right $\mathrm{AD}$ was $(1.24 \pm 0.80) \mathrm{mm}$ $(0.05-3.25 \mathrm{~mm})$; the $\mathrm{AD}$ of all specimens was $(1.26 \pm 0.97) \mathrm{mm}(0.00-4.60$ $\mathrm{mm})$. The left and right data were not normally distributed. The Wilcoxon test showed that there was no statistical difference $(P=0.33, P>0.05)$.

The horizontal distance AE between the center of the left mental foramen and the anterior median line was $(23.51 \pm 1.88) \mathrm{mm}(19.80-28.40 \mathrm{~mm})$; the right $\mathrm{AE}$ was $(23.64 \pm 1.77) \mathrm{mm}(20.80-28.90 \mathrm{~mm})$; the AE of all specimens was $(23.57 \pm$ $1.82) \mathrm{mm}(19.80-28.90 \mathrm{~mm})$. The left and right data were normally distributed. The paired sample $t$ test showed that there was no statistical difference $(\mathrm{P}=0.62$, $\mathrm{P}>0.05)$.

\section{Discussion}

The mental foramen and peripheral anatomical structures are of great importance to mental nerve block. The mental foramen is located on either side of the mandible where it is generally flat or slightly depressed. The inferior alveolar nerve divides into two branches in the mental foramen. One branch is small, extends forwards and controls the canines, lateral incisors, and central incisors. The other branch is large and called the mental nerve after passing the mental foramen, and divides into labial and mental branches that control the lower lip and the mental skin.

Operationally and clinically, the mental foramen cannot be easily located and found due to the small diameter, different sizes and different opening directions, as well as the presence of the submental foramen. In this study, in order to better guide the clinical operation, the morphological observation of the mental foramen and the measurement of relevant data were performed for mental nerve block. The morphology of 50 specimens (100 sides) of mental foramen was basically symmetrical. Circular mental foramina are the largest in number, accounting for $73 \%$, followed by oval mental foramina, accounting for $27 \%$. No mental foramen of other shape was found. The morphological observation on the mental foramen was different from the observations by other scholars at home and abroad [2] [3]. The observations of most scholars showed that oval mental foramina are the most in mumber, followed by the circular ones, and that there are irregular mental foramina. One of the reasons for this may be that there is a difference in the mental foramen among different regions, races, and nationalities. The other reason may be due to the small sample size observed in this study, or the use of different observation method. The outward and upward opening with an angle of about $45^{\circ}$ of the mental foramen is common, which is basically consistent with the study data of Li Yuehong [4] et al. The opening direction indicates that the needle should be inserted from the top-rear up to the 
bottom-front to find the mental foramen during mental nerve block. According to the observation data, mental foramina below the first premolar account for 20\%; those below the second premolar account for $75 \%$; and those below the first molar account for 5\%, indicating that most mental foramina are below the first and the second premolars. The results of many similar literature data reports were basically the same as those of this study, showing no regional, racial, and gender differences [5] [6]. Considering the opening direction of mental foramen, the entry point should be in the posterior lower part of the second premolar during mental nerve block, so that the needle tip can better reach the mental foramen. The occurrence rate of submental foramen is $1 \%$, which is much lower than other observation data. Emre [7] observed via CBCT that the occurrence rate of submental foramen in 1005 patients was $12.23 \%$. Wang Tingting [8] et al. in China also observed via CBCT that the occurrence rate of submental foramen in 500 patients was $7.8 \%$. The difference may be caused by the different observed objects and measurement methods. The occurrence rate of submental foramen in this study is close to the result of Meng Song's observation, i.e., $0.43 \%$ [9]. The sample size should be further increased in follow-up studies, so as to obtain more accurate data. In case of the poor effect of mental nerve block, the presence of submental foramen should be considered; and a double-hole block is required during the treatment. Three sets of data were measured in the sagittal position of the mental foramen. Such data indicated that the center of the mental foramen is basically $1.26 \mathrm{~mm}$ above the center of the superior and inferior borders of mandible. Some research scholars believed that the position of the mental foramen is correlated to age [10]. With the increase in age, the mental foramen gradually moves upwards and backwards. For toothless elderly, the position of the mental foramen may be slightly inclined to the superior border of the mandible due to the absorption of the mandibular alveolar bone. Due to the selection of specimens in this study, the measurement data were quite consistent with this point of view. Therefore, in clinical practices, the entry point during mental nerve block is preferably above the horizontal midline of the mandible. The set of data finally measured is the horizontal distance from the center of the mental foramen to the anterior median line, which was $23.57 \pm$ $1.82 \mathrm{~mm}$. Some observation data indicated that the distance from the mental foramen to the anterior median line in men is slightly longer than that in women [11]. During clinical operation, the opening direction of the mental foramen is still considered. An opening $2.5 \mathrm{~cm}$ from the median line is recommended for nerve block.

B-ultrasound has been more and more widely used to guide clinical operation in mental nerve block. According to the observations in this study, for B-ultrasound-guided mental nerve block, in-plane needle insertion is recommended. A small linear array probe is placed below the second premolar with an opening $2.5 \mathrm{~cm}$ from the median line, tilting outwards and upwards. The probe moves from the head to the tail until the mandibular discontinuity, i.e. the men- 
tal foramen, is visible. During nerve block, attention should be paid not to damage the blood vessels with the needle tip.

This study provided the scientific data for the clinical research on the mental foramen, but there still are many imperfections, such as the number of the specimens was insufficient, the means of measurement were not advanced enough and wider areas should be included in this study.

\section{Conclusion}

This study confirmed that the entry point for mental nerve block should be in the lower back part of the second premolar and above the horizontal midline of the mandible, $2.5 \mathrm{~cm}$ from the anterior median line. The needle should be inserted forwards and downwards to find the mental foramen.

\section{Conflicts of Interest}

The authors declare no conflicts of interest regarding the publication of this paper.

\section{References}

[1] Osama, H., Ackerman, R.S., Trip, E., et al. (2018) Mental Nerve Blocks for Lip Brachytherapy: A Case Report. A \& A Case Reports, 10, 265-266. https://doi.org/10.1213/XAA.0000000000000681

[2] Li, Y.M., Chen, K.G., Han, S.Y., et al. (2009) Observation on Mental Foramen of Chinese People. Heilongjiang Medical Science, 32, 18-19.

[3] Rajoria, S.R. and Chauhan, S. (2018) Study of Position, Shape and Size of Mental Foramen in Dry Adult Human Mandibles at S.M.S. Medical College, Jaipur. Journal of the Anatomical Society of India, 67, 41-41. https://doi.org/10.1016/j.jasi.2018.06.049

[4] Li, Y.H., Zhao, Z., Jia, J., et al. (2019) Measurement and Analysis of CBCT in 200 Adults with Mental Foramen in Urumqi Area. China Cosmetic Medicine, 28, 95-98.

[5] von Arx, T., Friedli, M., Sendi, P., et al. (2013) Location and Dimensions of the Mental Foramen: A Radiographic Analysis by Using Cone-Beam Computed Tomography. Journal of Endodontics, 39, 1522-1528. https://doi.org/10.1016/j.joen.2013.07.033

[6] Cao, K., Li, J.F., Bao, Q., et al. (2017) Measurement and Analysis of Mental Foram Area Based on Conical Beam CT. Journal of Clinical Stomatology, 33, 303-305.

[7] Aytugar, E., Özeren, C., Lacin, N., et al. (2019) Cone-Beam Computed Tomographic Evaluation of Accessory Mental Foramen in a Turkish Population. Anatomical Science International, 94, 257-265. https://doi.org/10.1007/s12565-019-00481-7

[8] Wang, T.T., Tang, Y.C. and Wang. Y.Q. (2016) Measurement and Analysis of Conical Beam CT for Submental Foramina. Journal of Clinical Practice Hospital, 13, 98-100.

[9] Meng, S., Lu, C.Q. and Zou, D.R. (2019) Cone-Beam CT Analysis of the Morphology and Position of Mandible Mental Foramen. Journal of Modern Stomatology, 33, 95-97.

[10] Yan, Y.F. and Meng, X.Y. (2009) Progress in Anatomical Structure of Mental Fora- 
men. Journal of Clinical Rational Drug Use, 2, 90-92.

[11] Fang, H.B. and Wang, B. (2016) Measurement and Analysis of the Position of Mental Foramen in Normal People. Journal of Oral and Maxillofacial Surgery, 26, 435-437. 